Mariño-Fernández, R., Barreria-Cerqueiras, E.M., Rego-Agraso, L. \& Irmscher, M. (2021). La formación inicial y continua del cuerpo docente de FP: satisfacción y competencia percibida en tiempos de crisis. Revista Electrónica Interuniversitaria de Formación del Profesorado, 24(2), 193-209.

DOI: https://doi.org/10.6018/reifop.470391

\title{
La formación inicial y continua del cuerpo docente de FP: satisfacción y competencia percibida en tiempos de crisis
}

Raquel Mariño Fernández ${ }^{(1)}$, Eva M. Barreira Cerqueiras ${ }^{(1)}$, Laura Rego-Agraso ${ }^{(2)}$ y Maike Irmscher ${ }^{(3)}$

(1) Universidade de Santiago de Compostela; ${ }^{(2)}$ Universidade da Coruña; ${ }^{(3)}$ Carl Von Ossietzky Universität Oldenburg- Alemania

\section{Resumen}

Este trabajo pretende analizar el nivel de satisfacción del profesorado de Formación Profesional con la formación pedagógica inicial recibida, así como el nivel de adquisición de las competencias docentes y la valoración de la formación continua realizada una vez que ejercen activamente su profesión. También pretende conocer los ámbitos sobre los que demandan acciones formativas vs aquellos que les oferta la Administración, y los elementos que se consideran facilitadores o limitadores para involucrarse en dichas acciones. El enfoque empírico se basa en una encuesta telemática desarrollada con un cuestionario elaborado ad hoc $(\alpha=.899)$ y cumplimentado por una muestra de 245 profesores/as de FP de la comunidad autónoma de Galicia (España). Como principales resultados observamos que la formación continua es ligeramente mejor valorada que la formación pedagógica inicial, así como que los ámbitos sobre los que se demanda formación continua coinciden parcialmente con los que oferta, a juicio de los/as docentes, la Administración educativa. Asimismo, destacan la excesiva burocracia asociada a las tareas cotidianas y los horarios docentes sobrecargados, como dos de los principales limitadores de la participación del profesorado en acciones de formación continua.

\section{Palabras clave}

Formación continua; formación pedagógica inicial; formación profesional; profesorado.

\section{Contacto:}

Raquel Mariño Fernández, raquel.marino@usc.es, Facultad de Ciencias de la Educación-Campus Vida Despacho 76 (módulo C2) USC, 15786 Santiago de Compostela. 


\title{
The initial and continuous training of vocational teachers: satisfaction and perceived competence in times of crisis
}

\begin{abstract}
This work studies the elements present in the current model of initial and continuous training of Vocational Training teachers. It aims to analyze the level of satisfaction of vocational teachers with the initial pedagogical training received, the level of acquisition of teaching skills, as well as the continuous training received once they achieve a position as vocational teacher. It also aims to know the areas on which continuous training actions are required $v s$ those offered by the Educational Administration, and the elements that are considered facilitators or limiters to get involved in these continuous training actions. The empirical approach is based on a telematic survey developed with an ad hoc questionnaire $(\alpha=.899)$ and completed by a sample of 245 VET teachers from the autonomous community of Galicia (Spain). As main results, we observe that continuous training is slightly better valued than initial pedagogical training, as well as the areas in which continuous training is demanded partially coincide with those offered, in the opinion of teachers, by the Educational Administration. Likewise, they highlight the excessive bureaucracy associated with daily tasks and overloaded teaching schedules, as two of the main limiters of the participation of teachers in continuous training actions.
\end{abstract}

\section{Key words}

Continuous training; initial pedagogical training; vocational and educational training (VET); teachers.

\section{Introducción}

Los diferentes estudios sobre formación del profesorado recogen, mayoritariamente, dos líneas de estudio: los que ponen el foco de atención en la Formación Inicial (de aquí en adelante, $\mathrm{FI}$ ) —definida como la que ofrece el sistema educativo formal y con la que se trata de preparar a los individuos para el desempeño de una profesión-(Bolívar, 2007; Imbernón y Colén, 2015; Sarceda-Gorgoso, Santos-González y Rego-Agraso, 2020) y los que ponen el foco de atención sobre la Formación Continua (de aquí en adelante, FC) —definida como herramienta de mejora de la cualificación profesional al servicio del desempeño laboral(Esperanza, 2006; Escudero Muñoz, 2020).

También han sido frecuentes los trabajos que abordan las particularidades de los cuerpos docentes de Educación Primaria y Educación Secundaria Obligatoria (ESO) centrados en identificar los factores actitudinales, motivacionales y/o vocacionales (Perrenoud, 2010; Urkidi, Losada, López y Yuste,2015; Prats, 2016; Muñoz-Fernández, Rodríguez-Gutiérrez y Luque-Vilchez, 2019), la valoración de las competencias docentes, niveles de cualificación y acceso a dichos cuerpos docentes (Tejada Fernández, 2009; Rial Sánchez, 2010; Valle y Manso,2014; Mas-Torelló, 2016; Eurydice, 2018; Sarceda-Gorgoso y Rodicio-García, 2018; Sarceda-Gorgoso, Santos González y Rego Agraso, 2020), la evolución de dimensiones referidas al marco legislativo y políticas de actualidad - a nivel estatal y europeo- con estudios comparados (Senent, 2011; Tiana, 2013; Ayala y Luzón, 2013; Fernández-Berrueco y Sánchez-Tarazaga, 2014; Lorenzo-Vicente, Muñoz-Galiano y Beas-Miranda, 2015) o sobre 
metodologías, estrategias y retos docentes aún sin alcanzar (Martínez y Prats, 2018; Martínez, 2016; UNESCO, 2015; López Rupérez, 2013).

No obstante, son pocas las investigaciones que dirigen su atención principal al estudio de la formación del cuerpo docente de Formación Profesional en sus dos vertientes -inicial y continua- La intencionalidad del presente trabajo se centra precisamente en conocer esta realidad tan poco explorada y contribuir, con sus resultados, al desarrollo de mejoras formativas y avance del conocimiento científico en el ámbito docente de la Formación Profesional (de aquí en adelante, FP).

Partimos de que la FI del cuerpo docente asienta sus bases curriculares sobre la necesidad de adquisición de competencias didácticas y pedagógicas básicas que les permitirán impartir docencia. En cambio, la FC permite cubrir las necesidades formativas, laborales y de recualificación que les van demandando los entornos profesionalizantes a los profesionales (Molina y Esteve, 2016; Serrano y Pontes, 2017), permaneciendo sujeta a los cambios económicos, sociales y culturales que le afectan. Hoy en día la FC no se concibe desligada de las organizaciones laborales ni de las instituciones educativas, por varias razones:

- Se considera la herramienta más eficaz para gestionar y readaptarnos a los continuos, vertiginosos y convulsos cambios que se vienen sucediendo en las sociedades tecnológicas actuales (Fundación Tripartita para la formación y el empleo, 2003; Pineda, 2007; OCDE, 2011; MECD, 2015).

- La inversión de capital en la formación de los asalariados/as se ha consolidado como un recurso productivo y competitivo.

- Se han afianzado estructuras propias, que se encargan de planificarla, ofertarla y gestionarla en función de las necesidades de los trabajadores/as y sectores profesionales.

- Se adapta a la revolución digital, ofreciendo nuevos servicios, eliminando barreras espaciotemporales y garantizando una formación más equitativa.

\section{La formación inicial y continua del profesorado de FP: marco legislativo}

Para contextualizar esta realidad es importante señalar que, en España, para ejercer la docencia en el sistema de FP es preciso contar con el título oficial de Máster de Profesorado de Educación Secundaria (MPES) que acredita la formación pedagógica y didáctica de acuerdo con lo exigido por los artículos 94, 95 y 97 de la Ley Orgánica 2/2006, de 3 de mayo, de Educación. El ejercicio de la profesión docente en Educación Secundaria Obligatoria y Bachillerato, Formación Profesional y Enseñanzas de Idiomas, está regulado por lo dispuesto en la Orden ECl/3858/2007, de 27 de diciembre, que establece los requisitos oficiales que habiliten para el ejercicio docente. Sin embargo, en la disposición adicional primera de la mencionada ley orgánica se indica que la formación pedagógica y didáctica del profesorado que, por razones derivadas del tipo de titulación inicial, no pueda adquirirse en estudios de Máster, será acreditada mediante una formación pedagógica equivalente. Esta salvedad se plantea para atajar la situación de aquellos profesionales que cuentan con una titulación de FP que no tiene ningún grado universitario asociado, pero que son necesarios como docentes en el sistema de FP, dado que sus titulaciones están presentes en el mismo'. Actualmente

\footnotetext{
${ }^{1}$ Nos referimos a los profesionales de las familias de (Real Decreto 276/2007): Imagen personal (Estética y peluquería); Hostelería y Turismo (Cocina, pastelería y servicios de restauración); Textil,
} 
existen cursos de especialización pedagógica y didáctica — desarrollados por diversas universidades a lo largo del territorio español- que permiten a estos profesionales adquirir las competencias que les den acceso al — hasta ahora reconocido- cuerpo docente de profesores/as técnicos de FP.

Por otra parte, la formación permanente del profesorado se regula a nivel autonómico, tomando como referente la actual Recomendación del Consejo Europeo del 22 de mayo de 2018 , relativa a las competencias clave para el aprendizaje permanente (2018/C 189/01) y la Orden EDU/2886/2011 por la que se regula la convocatoria, reconocimiento, certificación y registro de las actividades de formación permanente del profesorado. En este sentido, la normativa vigente en materia de formación continua del profesorado en Galicia es la Orden del 14 de mayo de 2013, por la que se regula la convocatoria, el reconocimiento, la certificación y el registro de actividades de formación permanente del profesorado en Galicia (DOG, 22/05/2013) y la Resolución del 23 de agosto de 2016, para la misma finalidad. Cabe mencionar, además, que la propia Administración Educativa autonómica elabora un Plan Anual de Formación del Profesorado, un Plan de Formación Permanente del Profesorado en Centros Educativos y el Plan de Formación Profesional, todos ellos gestionados mediante el Centro Autonómico de Formación e Innovación (CAFI).

\section{Metodología}

\subsection{Objetivos y método del estudio}

Este estudio tiene por objetivo general analizar cuál es la percepción y valoración que tiene el profesorado de FP sobre la FI y la FC recibida — desde una perspectiva pedagógica y una vez que ya ejercen activamente su profesión-. Así mismo, como objetivos específicos se han establecido los siguientes:

- Conocer el nivel de satisfacción alcanzado por el profesorado de FP con su formación pedagógica inicial y con la adquisición de competencias docentes.

- Analizar ese mismo nivel de satisfacción con la FC ofertada por la Administración Educativa de la que dependen.

- Conocer las temáticas generales de las acciones formativas demandadas por este profesorado - a través de los departamentos didácticos de los centroscontraponiéndolas con las ofertadas por la Administración.

- Conocer los factores que limitan la participación de este profesorado en las acciones de FC, a la vez que los factores facilitadores de la misma - siempre bajo los ojos del cuerpo docente.

Para alcanzarlos se empleó el método de encuesta en un estudio eminentemente cuantitativo y con pretensiones descriptivas.

\subsection{Población y muestra}

La muestra del estudio está compuesta por un total de 245 profesores/as de FP de la comunidad autónoma de Galicia (España), de los cuales, el 49,3\% son hombres y el 50,7\% mujeres. La edad media de la muestra es de 47,05 años y la moda de 50 años. En cuanto a la

Confección y Piel (Patronaje y confección); Transporte y Mantenimiento de Vehículos; Madera, mueble y corcho y Fabricación Mecánica y Artes y artesanías (Producción de artes gráficas). A estas familias profesionales se añaden, además, las enseñanzas deportivas de régimen especial (Orden EDU/2645/2011). 
familia profesional en la que imparten docencia, se ha obtenido representación de las 23 familias que se ofertan en el territorio gallego al alumnado, siendo Sanidad la que cuenta con un mayor número de profesores/as encuestados (10,1\%), seguida por Servicios Socioculturales y a la Comunidad $(8,9 \%)$ y Hostelería y Turismo $(7,6 \%)$.

En cuanto a su situación laboral, la amplia mayoría son funcionarios de carrera $(62,4 \%)$, seguidos por profesores/as interinos/as (24,1\%), substitutos/as (8,4\%) y profesores/as contratados en centros privados de FP $(5,1 \%)$. Con respecto al cuerpo al que pertenecen, la mayor parte de la muestra la conforman profesores/as de Educación Secundaria (49,2\%), seguidos por el profesorado técnico de FP $(45,8 \%)$ y por profesores/as de centros privados $(3,8 \%)$. La media de experiencia profesional docente - contabilizando experiencia en el sector público y privado- es de 13,66 años, pero la moda es de 3 años.

En su mayoría (63\%), señalan que la titulación más alta que poseen es un Grado Universitario o equivalente —Diplomatura, Ingeniería técnica, Ingeniería o Licenciatura-, mientras que un $12,2 \%$ señala poseer, además, un título de Máster. Existe un 12,2\% que cuenta con un ciclo formativo de grado superior como titulación más alta y tan sólo un $4,3 \%$ señala poseer un doctorado.

En relación con su formación pedagógica inicial, un $61,4 \%$ indica que posee el ya inexistente Curso de Adaptación Pedagógica (CAP), seguido por el 15,9\% que señala tener el Máster en Profesorado de ESO, Bachillerato, FP y Enseñanza de Idiomas (MFPES). Del mismo modo, un $10,9 \%$ indica no contar con ninguna titulación pedagógica específica y un $5,5 \%$ señala que ha realizado el Curso de Especialización en Formación Pedagógica y Didáctica del Profesorado Técnico de Formación Profesional.

\subsection{Instrumento y aplicación}

El instrumento de recogida de datos empleado es un cuestionario elaborado ad hoc, tomando como referencias iniciales para su elaboración los trabajos de Sarceda Gorgoso (2002), Barreira Cerqueiras (2015) y USC (2020). Más concretamente, para formular los ítems referidos a las dimensiones vinculadas con el perfil docente, la tipología, características y elementos presentes en la $\mathrm{FI}$ y $\mathrm{FC}$ ofertada/cursada para el profesorado de $\mathrm{FP}$, se toma como referente principal el instrumento de Barreira Cerqueiras (2015). Igualmente, el trabajo de Sarceda Gorgoso (2002) ha servido para formular los ítems vinculados a las dimensiones de identificación de la diversidad de temáticas ofertadas/demandadas en la FC—bien desde los departamentos de su especialidad bien desde las entidades administrativas-, así como las referidas a los factores que las incentivan/dificultan. Finalmente, para formular los ítems referidos al nivel de satisfacción alcanzados con dichos procesos formativos, así como los de valoración de necesidades formativas en materia de competencias pedagógicas y/o de formación técnica vinculadas con su especialidad, nos hemos basado en el instrumento oficial de evaluación de la actividad docente de la propia USC (2020).

El cuestionario resultante, de respuesta anónima, queda compuesto por un total de 114 variables, distribuidas en 27 ítems de distinta tipología: preguntas cerradas de respuesta múltiple, preguntas dicotómicas (Sí/No), escalas tipo Likert de 4 opciones de respuesta y preguntas de respuesta abierta. El alfa de Cronbach para este instrumento se sitúa en ( $a$ =.899), por lo que podemos afirmar que se trata de un instrumento con consistencia interna.

Se optó por un diseño on line empleando la aplicación de Microsoft Forms para tal fin. Una vez diseñado se distribuyó a los centros de FP — públicos y privados- de la comunidad autónoma de Galicia (España) —mediante correo electrónico-y solicitando a los equipos directivos el traslado al profesorado de FP de su centro para su cumplimentación. 


\subsection{Análisis de datos}

Se realizó un tratamiento estadístico de los datos, empleando para ello el programa IBM Statistics versión 24. Se extrajeron así los estadísticos descriptivos de cada una de las variables - media, desviación típica (DT) y varianza-, así como las frecuencias de respuesta a cada una de ellas en porcentaje. Además, se calculó el alfa de Cronbach para determinar la fiabilidad de las escalas que componen el cuestionario.

Los resultados se han tratado con la confidencialidad que establece la Ley Orgánica 3/2018, de 5 de diciembre, de Protección de Datos Personales y garantía de los derechos digitales.

\section{Resultados}

En base a los resultados obtenidos, la media de satisfacción global con la formación pedagógica inicial del profesorado de FP es de un 2,38 (DT 0,754). Tal como podemos apreciar en la tabla 1, los elementos mejor valorados en la $\mathrm{Fl}$ recibida por el profesorado que actualmente están en ejercicio son los horarios académicos $(2,75)$ y los espacios/aulas $(2,64)$. Le siguen los docentes que impartieron la formación $(2,57)$, la duración total $(2,54)$ y la evaluación desarrollada $(2,50)$. Por el contrario, los elementos peor valorados son las metodologías implementadas $(2,31)$, los recursos y materiales empleados $(2,32)$ y los contenidos abordados $(2,36)$.

Tabla 1.

Nivel de satisfacción con la formación pedagógica inicial (Media, Desviación Típica y Varianza). Fuente: Elaboración propia

\begin{tabular}{lccc}
\hline $\begin{array}{l}\text { Nivel de satisfacción con la formación pedagógica } \\
\text { inicial }\end{array}$ & Media & DT & Varianza \\
\hline Duración total & 2,54 & 0,815 & 0,665 \\
\hline Docentes que imparten la formación & 2,57 & 0,774 & 0,600 \\
\hline Contenidos abordados & 2,36 & 0,751 & 0,564 \\
\hline Metodologías implementadas & 2,31 & 0,801 & 0,641 \\
\hline Espacios/Aulas & 2,64 & 0,783 & 0,613 \\
\hline Horarios académicos & 2,75 & 0,795 & 0,631 \\
\hline Recursos y materiales didácticos empleados & 2,32 & 0,792 & 0,627 \\
\hline Evaluación desarrollada & 2,50 & 0,772 & 0,596 \\
\hline Satisfacción global & 2,38 & 0,754 & 0,568 \\
\hline
\end{tabular}

Con respecto al desarrollo de las competencias docentes facilitada por la formación pedagógica inicial cursada (ver tabla 2), podemos destacar que, en general, son puntuaciones con tendencia a la baja — todas ellas por debajo de 2,5-, siendo peor valoradas las competencias relacionadas con el desarrollo de contenidos curriculares especializados $(1,94)$, la atención a la diversidad $(1,99)$ y el diseño y usos de recursos didácticos, digitales y de innovación (2). 
Tabla 2.

Valoración respecto de si la formación pedagógica cursada le facilitó el desarrollo de competencias relacionadas con los siguientes ámbitos del ejercicio docente (Media, Desviación Típica y Varianza). Fuente: Elaboración propia

\begin{tabular}{lccc}
\hline $\begin{array}{l}\text { Valoración adquisición competencias docentes en la } \\
\text { formación pedagógica inicial }\end{array}$ & Media & DT & Varianza \\
\hline Procesos de enseñanza-aprendizaje en la FP & 2,03 & 0,865 & 0,748 \\
\hline Contenidos curriculares especializados & 1,94 & 0,817 & 0,668 \\
\hline $\begin{array}{l}\text { Programación de la enseñanza en el aula y en el } \\
\text { entorno }\end{array}$ & 2,09 & 0,841 & 0,707 \\
\hline $\begin{array}{l}\text { Diseño y usos de recursos didácticos, digitales y de } \\
\text { innovación }\end{array}$ & 2 & 0,8 & 0,640 \\
\hline $\begin{array}{l}\text { Metodologías didácticas } \\
\text { Atención en la diversidad }\end{array}$ & 2,18 & 0,801 & 0,642 \\
\hline $\begin{array}{l}\text { Fomento de la equidad, accesibilidad, respeto y } \\
\text { sostenibilidad }\end{array}$ & 2,03 & 0,838 & 0,703 \\
\hline $\begin{array}{l}\text { Información, orientación y asesoramiento del } \\
\text { alumnado }\end{array}$ & 2 & 0,794 & 0,631 \\
\hline
\end{tabular}

En cuanto a la FC ofertada por la Administración Educativa, vemos que el nivel de satisfacción aquí es algo más elevado que el señalado para la FI (ver tabla 3). En este caso, las medias se sitúan todas ellas por encima del 2,5, siendo los/as docentes que imparten la formación el elemento mejor valorado $(3,17)$, seguido de los contenidos abordados $(3,05)$ y de la evaluación desarrollada (3).

Tabla 3.

Nivel de satisfacción con la formación continua a la que se ha asistido en el ejercicio docente (Media, Desviación Típica y Varianza). Fuente: Elaboración propia

\begin{tabular}{lccc}
\hline $\begin{array}{l}\text { Nivel de satisfacción con la } \\
\text { formación continua ofertada por la } \\
\text { Administración }\end{array}$ & Media & DT & Varianza \\
\hline Duración total & 2,86 & 0,6 & 0,360 \\
\hline Docentes que imparten la formación & 3,17 & 0,671 & 0,451 \\
\hline Contenidos abordados & 3,05 & 0,668 & 0,446 \\
\hline Metodologías implementadas & 2,92 & 0,753 & 0,567 \\
\hline Espacios/Aulas & 2,98 & 0,71 & 0,505 \\
\hline Horarios académicos & 2,86 & 0,761 & 0,579 \\
\hline Recursos y materiales didácticos & 2,9 & 0,716 & 0,512 \\
\hline Evaluación desarrollada & 3 & 0,676 & 0,456 \\
\hline
\end{tabular}


En la comparativa entre las temáticas de FC demandadas por los departamentos y la FC ofertada por la Administración educativa (ver tabla 4), vemos que, mientras los departamentos siempre $(41,2 \%)$ o con frecuencia $(35,8 \%)$ demandan formación específica vinculada a su especialidad, también es la que el profesorado percibe como menos ofertada —un $56 \%$ señala que se oferta sólo alguna vez y un 10,1\% indica que nunca se oferta-. Esta situación puede observarse igualmente si comparamos las medias obtenidas en cada una de las escalas, donde la relativa a la demanda por parte de los Departamentos $(3,11)$ es mayor que la obtenida en la frecuencia ofertada por la Administración (2,32), siendo este tipo de formación junto con la vinculada al desarrollo sostenible y la educación ambiental $(2,12)$, las que menos se ofertarían a ojos del profesorado encuestado. Asimismo, esta última temática coincide con las menos demandadas por parte del profesorado $(1,59)$.

Contrastan también la formación en idiomas, que se percibe como más ofertada por la Administración $(2,90)$ que demandada por los Departamentos $(1,70)$. Algo semejante ocurre con la formación de equipos directivos (1,37 frente a 2,51), convivencia e inclusión (1,59 frente a 2,63) e igualdad, coeducación y prevención de la violencia de género (1,64 frente a 2,74).

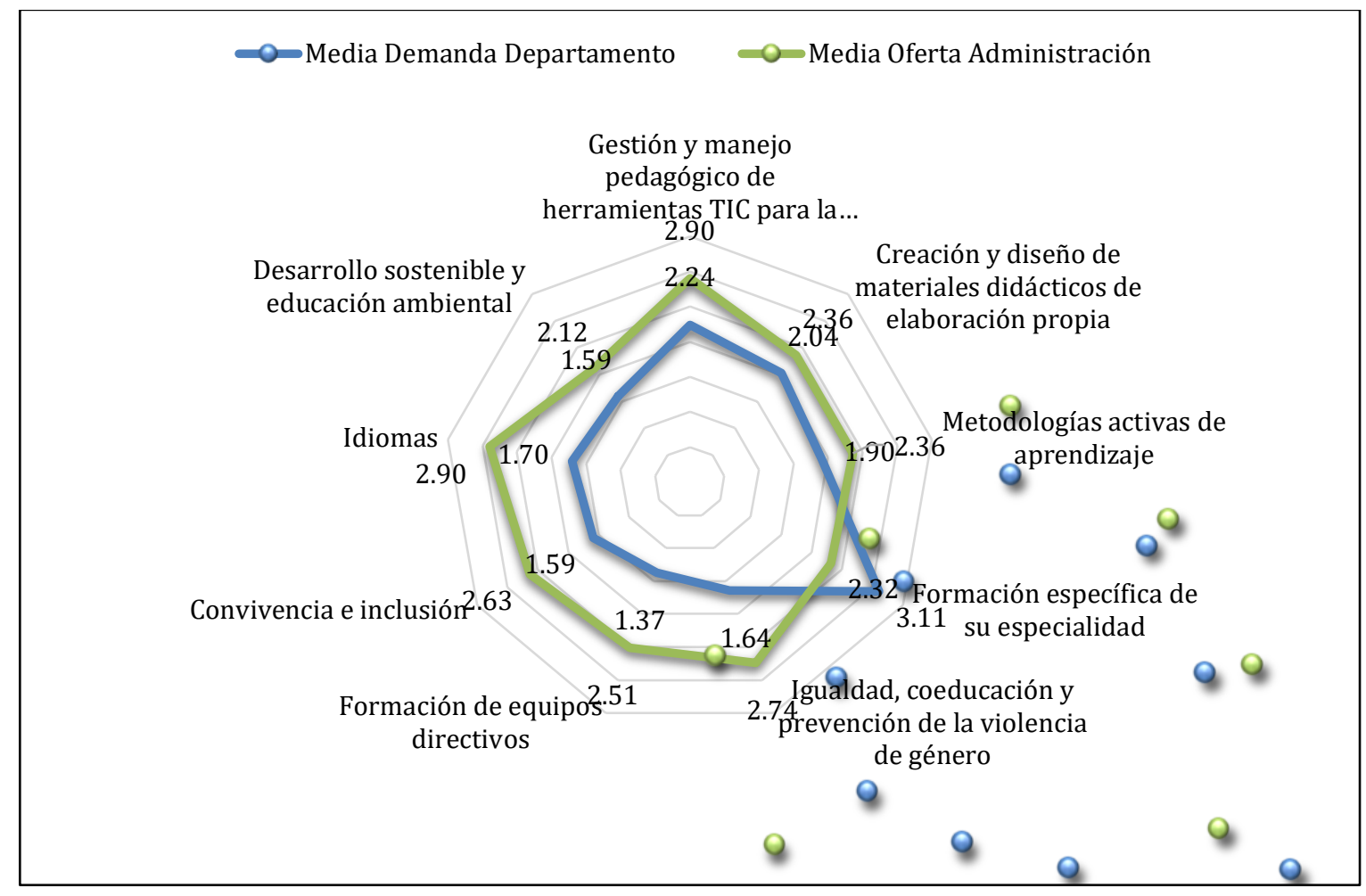

Figura 1. Comparativa de la media de FC ofertada por la Admón. Vs. media de FC demandada por Dpto. Fuente: Elaboración propia

En relación con los elementos que dificultan su participación en acciones de FC, cabe destacar dos aspectos clave: 1) la excesiva burocracia asociada a su labor docente cotidiana - como vemos en la figura 2 , el $81,2 \%$ de la muestra indica estar de acuerdo o muy de acuerdo con esta afirmación- y 2) la existencia de un horario docente semanal sobrecargado —el 75,3\% señala estar de acuerdo o muy de acuerdo con que ese hecho representa una dificultad en el acceso a la FC-. 


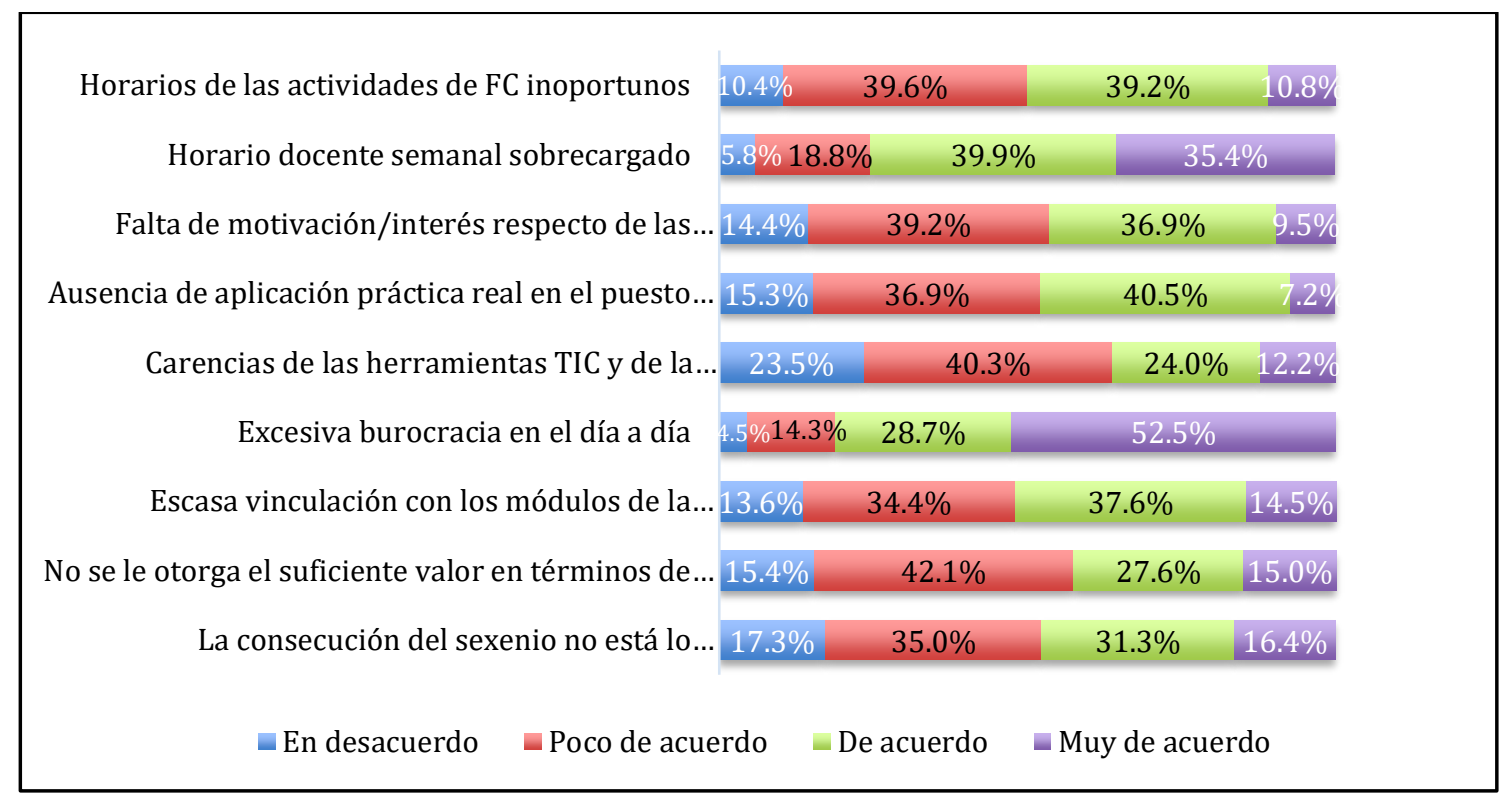

Figura 2. Grado de acuerdo con que las siguientes afirmaciones dificultan el acceso del profesorado a la formación continua (\%). Fuente: Elaboración propia

Si analizamos esta misma variable desde el punto de vista de las medidas de tendencia central (ver tabla 4), podemos apreciar como esas mismas categorías - horario docente semanal sobrecargado y excesiva burocracia en el día a día- obtienen las puntuaciones más altas (3,05 y 3,29, respectivamente). El siguiente elemento limitador más puntuado es la escasa vinculación de la formación con los módulos de la especialidad docente $(2,53)$, seguido por los horarios de las actividades de FC inoportunos $(2,5)$ y la insuficiente retribución del complemento de formación docente (sexenio) $(2,47)$.

Tabla 4.

Grado de acuerdo con que las siguientes afirmaciones dificultan el acceso del profesorado a la formación continua (Media, Desviación Típica y Varianza). Fuente: Elaboración propia

\begin{tabular}{lccc}
\hline Limitadores de la FC & Media & DT & Varianza \\
\hline Horarios de las actividades de FC inoportunos & 2,50 & 0,822 & 0,676 \\
\hline Horario docente semanal sobrecargado & 3,05 & 0,881 & 0,777 \\
\hline $\begin{array}{l}\text { Falta de motivación/interés respecto de las temáticas } \\
\text { ofertadas }\end{array}$ & 2,41 & 0,851 & 0,723 \\
\hline Ausencia de aplicación práctica real en el puesto de trabajo & 2,40 & 0,832 & 0,693 \\
\hline $\begin{array}{l}\text { Carencias de las herramientas TIC y de la conectividad para } \\
\text { realizar la formación }\end{array}$ & 2,25 & 0,952 & 0,906 \\
\hline Excesiva burocracia en el día a día & 3,29 & 0,875 & 0,766 \\
\hline Escasa vinculación con los módulos de la especialidad docente & 2,53 & 0,902 & 0,814 \\
\hline $\begin{array}{l}\text { No se le otorga el suficiente valor en términos de puntuación } \\
\text { para los concursos de profesorado }\end{array}$ & 2,42 & 0,925 & 0,855 \\
\hline
\end{tabular}




\begin{tabular}{lcccc}
\hline Limitadores de la FC & Media & DT & Varianza \\
\hline $\begin{array}{l}\text { La consecución del sexenio no está lo suficientemente } \\
\text { retribuido }\end{array}$ & 2,47 & 0,962 & 0,926 \\
\hline
\end{tabular}

Por último, con respecto a aquellos elementos que facilitarían el acceso a acciones de FC (ver tabla 5), la existencia de espacios formativos próximos que permitan conciliar es la categoría que recibe una puntuación más alta $(3,33)$. También tiene una puntuación elevada la necesidad de un mayor apoyo por parte de la Administración y los centros a la FC $(3,31)$ y mayor flexibilidad en los horarios académicos $(3,25)$. Los elementos que presentan una puntuación más baja —aunque superior a 2,5 en ambos casos-, son una mejor valoración en los concursos de traslados $(2,93)$ y la reducción de la ratio profesor/a-alumno/a $(3,17)$.

Tabla 5.

Grado de acuerdo con que las siguientes afirmaciones facilitan el acceso del profesorado a la formación continua (Media, Desviación Típica y Varianza). Fuente: Elaboración propia

\begin{tabular}{lccc}
\hline Facilitadores de la FC & Media & Desv. Típica & Varianza \\
\hline Reducción carga horaria semanal docente & 3,19 & 0,879 & 0,772 \\
\hline Mayor flexibilidad de horarios académicos & 3,25 & 0,809 & 0,654 \\
\hline $\begin{array}{l}\text { Mayor apoyo de la Administración y los centros } \\
\text { educativos }\end{array}$ & 3,31 & 0,751 & 0,563 \\
\hline $\begin{array}{l}\text { Espacios formativos próximos que permitan conciliar } \\
\text { Acceso a mejores recursos tecnológicos y de }\end{array}$ & 3,33 & 0,714 & 0,510 \\
$\begin{array}{l}\text { conectividad } \\
\text { Reducción de la ratio profesor/a-alumno/a }\end{array}$ & 3,17 & 0,783 & 0,614 \\
\hline Ampliación de la diversidad de la oferta formativa & 3,24 & 0,745 & 0,556 \\
\hline Mejor valoración en los concursos de traslados & 2,93 & 0,950 & 0,902 \\
\hline
\end{tabular}

\section{Discusión y conclusiones}

En este trabajo se identifican varios ejes de análisis y reflexión a partir de los datos recogidos sobre la formación pedagógica inicial y las competencias profesionales adquiridas del profesorado de FP, así como sobre el nivel de satisfacción con la formación continua ofertada, las temáticas más demandadas y los elementos facilitadores o limitadores de su participación en esta.

Sobre el nivel de satisfacción del profesorado con su formación pedagógica inicial los resultados obtenidos muestran que esta obtiene una puntuación que supera el aprobado $(2,38)$, lo que se puede interpretar como coherente con otras investigaciones sobre el particular (Sarceda-Gorgoso, Santos-González y Rego-Agraso, 2020), donde por ejemplo los futuros docentes, tras cursar el MFPES, perciben un incremento de su desarrollo competencial entre medio y medio-alto. Los elementos que peor se valoran en este estudio en relación con la FI son las metodologías utilizadas, los contenidos y los recursos educativos, lo que coincide con la apreciación de Rodríguez-Gutiérrez, Muñoz-Fernández y Luque-Vílchez 
(2018, p.1) cuando afirma que en la Fl del profesorado de secundaria las dimensiones de carácter estructural (sociales, políticas, culturales y organizativas) no han recibido la importancia suficiente.

Con respecto a las competencias pedagógicas desarrolladas con la $\mathrm{FI}$, las peor valoradas con una puntuación de 2 o inferior- son el desarrollo de contenidos curriculares especializados -, elemento en el que el alumnado del MFPES menos cambios apreciaba antes y después de la formación según el estudio de Sarceda-Gorgoso, Santos-González y Rego-Agraso (2020). Le siguen como las cuestiones peor valoradas, la atención a la diversidad y el diseño y uso de recursos didácticos, digitales y de innovación. En relación a la atención a la diversidad, en el Informe TALIS 2018 (OCDE, 2019) se indica que, en el caso del profesorado de secundaria español en centros multiculturales, los directores/as consideran que sólo el $64 \%$ apoya actividades u organizaciones que incentivan la expresión de los alumnos de identidades de diversos grupos culturales y étnicos, un porcentaje más bajo que en otros países de la OCDE —-Singapur, Colombia o Nueva Zelanda por ejemplo-, dónde hasta un $80 \%$ del profesorado que trabaja en centros multiculturales afirma que en su centro se llevan a cabo actividades de este tipo ${ }^{2}$.

Por otro lado, la puntuación que obtiene la FC es algo más alta $(2,5)$ que la formación pedagógica del profesorado, siendo los/as docentes que imparten la formación, la evaluación desarrollada y los contenidos abordados, los elementos mejor valorados. Estos resultados coinciden con los aportados en el estudio de García-Ruíz y Castro Zubizarreta (2012) quienes ya en su momento pusieron de manifiesto el alto grado de satisfacción del profesorado con la formación permanente recibida y, por tanto, su elevada valoración de la FC.

El tipo de formación demandada y ofertada es uno de los elementos clave en la medida en que el profesorado destaca, de forma bastante clara, que existen temáticas con bastante oferta formativa, lo que conlleva que las puntuaciones entre esta y la demanda se acerquen - como es el caso de la formación relativa a metodologías activas de aprendizaje o la creación y desarrollo de materiales didácticos ${ }^{3}-$ y otros elementos sobre los que no se incide lo suficiente a ojos del profesorado por parte de la Administración educativa. La temática más demandada y —-según el profesorado—, menos ofertada, es aquella relacionada con los contenidos específicos de su especialidad. Manifiestan así, la necesidad de recibir una formación teórico-práctica más renovada y conectada con su realidad profesional, algo que como indican Cobacho Casas y Pons García (2006, p.244) es necesario, dado que el profesorado debe poseer creatividad, iniciativa y capacidad de motivar e ilusionar, además de habilidades o disposición al conocimiento de las aptitudes básicas de la formación profesional específica del perfil profesional correspondiente en el que impartan su docencia.

También existen otras temáticas como los idiomas, la igualdad, coeducación y prevención de la violencia de género, la formación de equipos directivos o aspectos sobre convivencia e inclusión, que son considerados por el profesorado como temas más ofertados por la Administración que demandados por los departamentos. En este sentido, tal y como indican Mariño Fernández y Rial Sánchez (2019), la oferta de este tipo de contenidos debe incentivarse para capacitar a las personas frente a las discriminaciones, desigualdades y vulnerabilidad, en relación con los derechos sociales y laborales. Se puede decir entonces,

\footnotetext{
${ }^{2}$ En España ese porcentaje de docentes es menor, pero es mucho mayor que el de países con mayor índice de diversidad, como Noruega, Dinamarca, Suecia o Finlandia, donde no pasa del 30 \% (OCDE, 2019, p.111). ${ }^{3}$ Cabe mencionar aquí que en la Comunidad Autónoma existe una convocatoria específica para la elaboración de materiales didácticos vinculados a la especialidad docente por parte del profesorado, en la que el profesorado solicitante puede contar con un periodo específico de tiempo sin docencia dedicado exclusivamente a la elaboración de dicho material.
} 
que se incrementa entre el profesorado en activo la percepción de que, en contraposición a lo establecido por Escudero Muñoz, González González y Rodríguez Entrena (2018), se está desarrollando una formación continua más centrada en el 'cómo' que en contenidos vinculados con valores o aspectos sociales.

Otro de los elementos clave que se extraen de este estudio es la determinación por parte del profesorado de los elementos que dificultan (limitadores) o facilitan (facilitadores) su participación en acciones de FC. En este sentido, los elementos facilitadores mejor valorados son la existencia de espacios formativos próximos que permitan conciliar —que recibe la puntuación más alta-, un mayor apoyo por parte de la Administración y los centros a la FC y una mayor flexibilidad de los horarios académicos, coincidiendo este factor con los hallazgos de Rodríguez, Pozuelos y García (2012). Por el contrario, actuarían como limitadores del acceso a la FC del profesorado, la excesiva burocracia asociada a su labor cotidiana, un horario docente semanal sobrecargado y la escasa vinculación de la formación con los módulos de la especialidad profesional. Este exceso de burocracia o tareas adjuntas a la docencia, así como la escasez de tiempo en el horario para poder involucrarse en escenarios de FC, también se ha reflejado en los estudios de Dopico (2013) e Iglesias Martínez, Lozano Cabezas y Roldán Solé (2018).

Esta investigación, planteada en el contexto gallego, ha supuesto una aproximación a la percepción de la formación inicial y continua por parte del profesorado de FP, una cuestión ampliamente abordada en otros estudios, pero con distintos protagonistas. Aunar ambas tipologías de formación en una misma investigación, así como plantear los aspectos que aquí se recogen - abordados de manera unilateral en otros estudios - ha supuesto obtener una panorámica nada desdeñable de la realidad formativa percibida del profesorado de $\mathrm{FP}$ - que posibilita propuestas de mejoras en este campo como es la adecuación de la formación a las necesidades de conciliación-, y situarse como una contribución que arroja luz sobre las particularidades del profesorado de Formación Profesional en nuestro país, en cuanto a la percepción de la formación pedagógica inicial y del tipo de formación continua que se oferta.

Sin embargo, el alcance de esta investigación podría ampliarse desarrollando, por ejemploy a modo de contraste con los resultados cuantitativos obtenidos-, un proceso de investigación complementario de corte cualitativo que permitiese profundizar más en cuestiones como las variables influyentes en aquellas temáticas formativas mejor valoradas y causas de esta valoración; medidas, acciones y agentes implicados en la formación del colectivo docente; incidencia de otras variables socioculturales que puedan influir en los elementos limitadores y facilitadores; identificación de factores que puedan condicionar la oferta de acciones de FC vinculadas a temáticas relacionadas con los Objetivos de Desarrollo Sostenible (ODS) de la Agenda $2030^{4}$ —igualdad, violencia machista, innovación educativa, estrategia digital o desarrollo sostenible-. De igual modo, el alcance de este estudio se vería mejorado con la implicación de más agentes educativos y con una ampliación de la zona geográfica objeto de estudio, bien extendiéndola a todo el territorio español, bien comparando la realidad de Galicia con otras regiones de otros países europeos, como por ejemplo Alemania. Todo ello con la finalidad de poder constatar la existencia de pautas convergentes o divergentes en los resultados y respuestas obtenidas en este estudio.

\footnotetext{
${ }^{4}$ Véase la página oficial de la ONU y su contenido sobre la Agenda 2030 o la reciente ley LOMLOE (2020) y su alusión a dichos objetivos de desarrollo sostenible (ODS).
} 


\section{Referencias}

Ayala, A. y Luzón, A. (2013). Retos y desafíos de la formación del profesorado en el siglo XXI: una visión comparada. Revista Española de Educación Comparada, 22, 9-17. https://doi.org/10.5944/reec.22.2013.9320

Ametller, J. y Codina, F. (Coord.) (2017). La mejora de la formación inicial de docentes. Reflexiones y propuestas. Recuperado de https://mif.cat/wpcontent/uploads/2017/01/ModeloFormativoES.pdf

Barreira Cerqueiras, E. M. (2016). Análisis de la formación continua en medianas y grandes empresas: el caso de la comunidad gallega (Tesis doctoral). Universidade de Santiago de Compostela, Santiago de Compostela.

Bolívar, A. (2007). La formación inicial del profesorado de Secundaria y su identidad profesional. ESE: Estudios sobre educación, 12, 13-30. Recuperado de https://revistas.unav.edu/index.php/estudios-sobre-educacion/article/view/24326

Cobacho Casas, F. y Pons García J. (2006). Jóvenes en desventaja y cohesión social: educación y futuro para todos. Revista de Educación, 341, 237-258

Dopico, E. (2013) Secuencias Educativas: el docente digitalizado y la percepción de la enseñanza. Revista Iberoamericana para la Investigación y el Desarrollo Educativo, 3 (6), 126-150.

Escudero Muñoz, J. M. (2020). Un cambio de paradigma en la formación continuada del profesorado: escenario, significados, procesos y actores. Qurriculum: Revista de Teoría, Investigación y Práctica Educativa, 33, 97-125. DOI: https://doi.org/10.25145/j.qurricul.2020.33.06

Escudero Muñoz, J. M.; González González, M. T. y Rodríguez Entrena, M. J. (2018). Los contenidos de la formación continuada del profesorado: ¿Qué docentes se están formando? Educación XX1, 21(1), 157-180, DOI: 10.5944/educXX1.20183

Esperanza, F. J. (2006). Formación y autoformación permanente del profesorado. Avances en supervisión educativa: Revista de la Asociación de Inspectores de Educación de España, 3. Recuperado de https://avances.adide.org/index.php/ase/article/view/217

Eurydice (2018). La profesión docente en Europa: Acceso, progresión y apoyo. Informe de Eurydice. Luxemburgo: Oficina de Publicaciones de la Unión Europea.

Fernández-Berrueco, R. y Sánchez-Tarazaga, L. (2014). Competencias docentes en Secundaria. Análisis de perfiles de profesorado. Relieve, 20(1), 1-20.

Fundación Tripartita para la formación y el empleo (2003). Consolidación y desarrollo de la Formación Continua en España. Retos para la Formación Continua y sus instituciones en un futuro próximo. Madrid: Fundación Tripartita para la Formación en el Empleo. Recuperado de https://biblioteca.ief.es/cgi-bin/koha/opacdetail.pl?biblionumber=60634yshelfbrowse_itemnumber $=69135$

García-Ruíz, R., y Castro Zubizarreta, A. (2012). La formación permanente del profesorado basada en competencias. Estudio exploratorio de la percepción del profesorado de Educación Infantil y Primaria. Educatio Siglo XXI, 30(1), 297-322. Recuperado de https://revistas.um.es/educatio/article/view/149251

Gairín Sallán, J. (2011). Formación de profesores basada en competencias. Bordón, 63(1), 93108. 
Iglesias Martínez, M.J., Lozano Cabezas, I. y Roldán Solé, I. (2018). La calidad e innovación educativa en la formación continua docente: un estudio cualitativo en dos centros educativos. Revista Iberoamericana de Educación, 77(1), 13-34

Imbernón, F. (1989). La formación inicial y la formación permanente del profesorado. Dos etapas de un mismo proceso. Revista interuniversitaria de formación del profesorado, 6 , 487-499.

Imbernón, F. (2017). Ser docente en una sociedad compleja. Barcelona: Grao.

Imbernón, F. y Colén, M. T. (2015). Los vaivenes de la formación inicial del profesorado. Una reforma siempre inacabada. Tendencias Pedagógicas, 25, 57-76. Recuperado de https://revistas.uam.es/tendenciaspedagogicas/article/view/160

Ley Orgánica 2/2006, de 3 de mayo, de Educación (BOE, 106, 04/05/2006)

Ley Orgánica 3/2018, de 5 de diciembre, de Protección de Datos Personales y garantía de los derechos digitales (BOE, 6/12/2018)

Ley Orgánica 3/2020, de 29 de diciembre, por la que se modifica la Ley Orgánica 2/2006, de 3 de mayo, de Educación (BOE, 340, 30/12/2020)

López Rupérez, F. (2013). Profesión y formación del profesorado de Educación Secundaria. En A. Castillo Arredondo (Coord.) El profesor de Educación Secundaria para el siglo XXI: Fundamentos para su formación (pp. 50-58). Madrid: UNED

Lorenzo-Vicente, J.A., Muñoz-Galiano, I.M. y Beas-Miranda, M. (2015). Modelos de formación inicial del profesorado de Educación Secundaria en España desde una perspectiva europea. Revista Complutense de Educación, 26(3), 741-757.

Martínez, M. (2016). La formación inicial de maestros: una responsabilidad compartida. Bordón. Revista de Pedagogía, 68(2), 9-16.

Martínez, M. y Prats, E. (Coord.) (2018). Mejora para la formación inicial para la docencia. Acciones Estratégicas. Recuperado de https://mif.cat/wpcontent/uploads/2018/10/AccionsEstratES.pdf

Mariño Fernández, R. y Rial Sánchez, A. (2019) Balance de las necesidades formativas demandadas por mujeres que cursan o que han cursado ciclos de FP en familias profesionales masculinizadas: el caso de Galicia. Revista Educar, 55(1), 251-72. DOI: https://doi.org/10.5565/rev/educar.890

Mas-Torelló, O. (2016). La influencia de la experiencia en las competencias investigadoras del profesor universitario. Revista Complutense de Educación, 27(1), 13-34. DOI:10.5209/revRCED.2016.v27.n1.44706

Ministerio de Educación, Cultura y Deporte (MECD) (2015). Plan estratégico de aprendizaje a lo largo de la vida. Madrid: Ministerio de Educación, Cultura y Deporte. Recuperado de https://sede.educacion.gob.es/publiventa/plan-estrategico-de-aprendizaje-a-lo-largode-la-vida/ensenanza/20676

Molina, M.Á. y Esteve, R.P. (2016). El pensamiento del alumnado respecto de la formación inicial en el Máster de Educación Secundaria. En M.T. Tortosa Ybáñez, S. Grau Company y J.D. Álvarez Terual (Coords.). XIV Jornadas de Redes de Investigación en Docencia Universitaria. Investigación, innovación y enseñanza universitaria: enfoques pluridisciplinares (pp. 2004-2016). Alacant: Universitat d'Alacant 
Muñoz-Fernández, G.A., Rodríguez-Gutiérrez, P. y Luque-Vílchez, M. (2019). La formación inicial del profesorado de educación secundaria en España: perfil y motivaciones del futuro docente. Educación XX1, 22(1), 71-92, DOI: 10.5944/educXX1.20007

Organización de Naciones Unidas (ONU) (2015). La Agenda 2030 para el Desarrollo Sostenible. New York: ONU. Recuperado de https://www.un.org/sustainabledevelopment/es/development-agenda/

Organización de las Naciones Unidas para la Educación, la Ciencia y la Cultura (UNESCO) (2015). Replantear la educación. ¿Hacia un bien común mundial? París: UNESCO. Recuperado de https://unesdoc.unesco.org/ark:/48223/pfooo0232697

Organización para la Cooperación y el Desarrollo Económico (OCDE) (2011). Preparándose para trabajar. Barcelona: Fundación Barcelona FP.

Organización para la Cooperación y el Desarrollo Económico (OCDE) (2019). TALIS 2018 Estudio Internacional de la Enseñanza y del Aprendizaje. Informe Español. Madrid: Ministerio de Educación y Formación Profesional. Recuperado de https://sede.educacion.gob.es/publiventa/descarga.action?f_codigo_agc=21168

Orden ECl/3858/2007, de 27 de diciembre, por la que se establecen los requisitos para la verificación de los títulos universitarios oficiales que habiliten para el ejercicio de las profesiones de Profesor de Educación Secundaria Obligatoria y Bachillerato, Formación Profesional y Enseñanzas de Idiomas (BOE, 312, 29/12/2007).

Orden del 14 de mayo de 2013, por la que se regula la convocatoria, el reconocimiento, la certificación y el registro de actividades de formación permanente del profesorado en Galicia (DOG, 22/05/2013).

Orden EDU/2645/2011 e 23 de septiembre, por la que se establece la formación equivalente a la formación pedagógica y didáctica exigida para aquellas personas que estando en posesión de una titulación declarada equivalente a efectos de docencia no pueden realizar los estudios de máster (BOE, 240, 05/10/2011).

Orden EDU/2886/2011, del 20 de octubre, por la que se regula la convocatoria, reconocimiento, certificación y registro de las actividades de formación permanente del profesorado (BOE, 28/10/2011).

Pereira, F. (2010). Childhood Narratives in Initial Teacher Training: Childhood Government and Its Re-Institutionalization. Research in Education, 83(1), 1-16.

Perrenoud, P. (2010). La formación del profesorado: un compromiso entre visiones inconciliables de la coherencia. Revista Interuniversitaria de Formación del Profesorado, 68, 103-122.

Pineda Herrero, P. (2007). La formación continua en España: Balance y retos de futuro. RELIEVE, $\quad 13 \quad$ (1), 43-65. Recuperado de http://www.uv.es/RELIEVE/v13n1/RELIEVEv13n1_2.htm

Prats, E. (2016). La formación inicial docente entre profesionalismo y vías alternativas: mirada internacional. Bordón, 68(2), 19-33.

Recomendación del Consejo Europeo del 22 de mayo de 2018, relativa a las competencias clave para el aprendizaje permanente (2018/C 189/01). Diario Oficial de la Unión Europea, $4 / 6 / 2018$. 
Real Decreto 276/2007 de 23 de febrero, por el que se aprueba el Reglamento de ingreso, accesos y adquisición de nuevas especialidades en los cuerpos docentes a que se refiere la Ley Orgánica 2/2006, de 3 de mayo, de Educación, y se regula el régimen transitorio de ingreso a que se refiere la disposición transitoria decimoséptima de la citada ley (BOE, 53, 02/03/2007).

Resolución del 23 de agosto de 2016, de la Dirección Xeral de Educación, FP e Innovación Educativa, por la que se regula el reconocimiento, la certificación y el registro de la participación del profesorado en proyectos europeos como actividades de formación permanente del profesorado (DOG, 31/08/2016).

Rodríguez-Gutiérrez, P., Muñoz-Fernández, G. A., y Luque-Vílchez, M. (2018). Estudio exploratorio sobre el panorama actual de la formación inicial del profesorado de secundaria en España. IJERI: International Journal of Educational Research and Innovation, 11, 169-184. Recuperado de https://www.upo.es/revistas/index.php/IJERI/article/view/2808

Rodríguez, F. P., Pozuelos, F. J. y García, F. J. (2012). Cuando el cambio llega a la escuela. Estudio de casos sobre los procesos de innovación educativa. Revista Electrónica de Investigación y Docencia (REID), 8, 117-141.

Rial Sánchez, A. (1997). La Formación Profesional: introducción histórica, diseño de currículum y evaluación. Santiago de Compostela: Tórculo.

Rial Sánchez, A. (2010). La planificación y el diseño curricular por competencias: un reto para la educación del futuro. Revista Electrónica de Desarrollo de Competencias (REDEC), 1(5), 29-41.

Sarceda-Gorgoso, M.C. (2002). Análisis de las necesidades formativas del profesorado de formación profesional en Galicia (Tesis doctoral). Universidade de Santiago de Compostela, Santiago de Compostela.

Sarceda-Gorgoso, M.C. y Rodicio-García, M.L (2018). Escenarios formativos y competencias profesionales en la formación inicial del profesorado. Revista Complutense de Educación, 29(1), 147-164.

Sarceda-Gorgoso, M.C., Santos-González, M.C. y Rego-Agraso, L. (2020). Las competencias docentes en la formación inicial del profesorado de educación secundaria. Profesorado. Revista de currículum y formación del profesorado, 24(3), 402-421, DOI: 10.30827/PROFESORADO.V24I3.8260

Senent, J. M. (2011). La formación inicial del profesorado de Educación Secundaria: una mirada comparativa en la Unión Europea. Edetania, 39, 71-87.

Serrano, R. y Pontes, A. (2017). Diferencias entre expectativas y logros en las competencias del Prácticum del Máster de Formación del Profesorado de Enseñanza Secundaria. Revista Electrónica Interuniversitaria de Formación del Profesorado, 20(1), 1-18.

Tejada Fernández, J. (2009). Competencias docentes. Profesorado, 13(2), 0-15. Recuperado de http://hdl.handle.net/10481/7373

Tiana, A. (2013). Los cambios recientes en la formación inicial del profesorado en España: una reforma incompleta. Retos y desafíos de la formación del profesorado en el siglo XXI: una visión comparada. Revista Española de Educación Comparada, 22, 39-58. 
Universidade de Santiago de Compostela (USC) (2020). Cuestionario para o estudante. Satisfacción coa docencia recibida. Recuperado de https://www.usc.es/export9/sites/webinstitucional/gl/goberno/vrcalidade/descargas/E NQUISAS2014/2011-12-

01_Cuestionario_SATISFACCIXN_COA_DOCENCIA_RECIBIDA.pdf

Urkidi, P., Losada, D., López, V. y Yuste, R. (2015). El Acceso a la Formación Inicial del Profesorado y la Mejora de la Calidad Docente. Revista Complutense de Educación, 31 (3), 353-364.

Valle. J. M. y Manso, J. (2014). La voz del profesorado. Acceso a la profesión docente e inserción en el puesto de trabajo. Madrid: Consejo General de los Ilustres ColegiosOficiales de Doctores y Licenciados en Filosofía y Letras y en Ciencias. Recuperado de http://www.consejogeneralcdl.es/images/LA\%20VOZ\%20DEL\%2OPROFESORADO.pdf

\section{Apéndice 1.}

Frecuencia con la que se oferta formación continua por parte de la Administración educativa y se demanda por parte de los Departamentos Didácticos por temáticas (Media, Desviación Típica y Varianza). Fuente: Elaboración propia

\begin{tabular}{l|c|c|c|c|c|c}
\hline & \multicolumn{2}{|c|}{$\begin{array}{c}\text { Frecuencia Departamento } \\
\text { demanda FC }\end{array}$} & \multicolumn{2}{c}{$\begin{array}{c}\text { Frecuencia Administración } \\
\text { Educativa ofrece FC }\end{array}$} \\
\hline $\begin{array}{l}\text { Gestión y manejo } \\
\text { pedagógico } \\
\text { herramientas TIC para } \\
\text { la docencia }\end{array}$ & 2,24 & 0,922 & 0,851 & 2,90 & 0,674 & 0,454 \\
\hline $\begin{array}{l}\text { Creación y diseño de } \\
\text { materiales didácticos } \\
\text { de elaboración propia }\end{array}$ & 2,04 & 0,893 & 0,798 & 2,36 & 0,802 & 0,643 \\
\hline $\begin{array}{l}\text { Metodologías activas } \\
\text { de aprendizaje }\end{array}$ & 1,90 & 0,850 & 0,723 & 2,36 & 0,772 & 0,597 \\
\hline $\begin{array}{l}\text { Formación específica } \\
\text { de su especialidad }\end{array}$ & 3,11 & 0,916 & 0,839 & 2,32 & 0,767 & 0,588 \\
\hline $\begin{array}{l}\text { lgualdad, coeducación } \\
\text { prevención de la } \\
\text { violencia de género }\end{array}$ & 1,64 & 0,861 & 0,742 & 2,74 & 0,805 & 0,648 \\
\hline $\begin{array}{l}\text { Formación de equipos } \\
\text { directivos }\end{array}$ & 1,37 & 0,738 & 0,545 & 2,51 & 0,831 & 0,690 \\
\hline $\begin{array}{l}\text { Convivencia e inclusión } \\
\text { Idiomas }\end{array}$ & 1,59 & 0,848 & 0,719 & 2,63 & 0,763 & 0,583 \\
\hline $\begin{array}{l}\text { Desarrollo sostenible y } \\
\text { educación ambiental }\end{array}$ & 1,59 & 0,781 & 0,610 & 2,12 & 0,669 & 0,447 \\
\hline
\end{tabular}

